\title{
A simulation tool to assess the lightning induced over-voltages on de cables of photovoltaic installations
}

\begin{abstract}
The development of large scale photovoltaic (PV) plants in rural areas is constantly increasing. This paper describes appropriately scaled laboratory tests and geometrically accurate (real scale) simulation models in an attempt to assess the induced over-voltages on long dc cabling loops.
\end{abstract}

Keyword: Lightning protection; Large scale PV plants; Dc cabling 